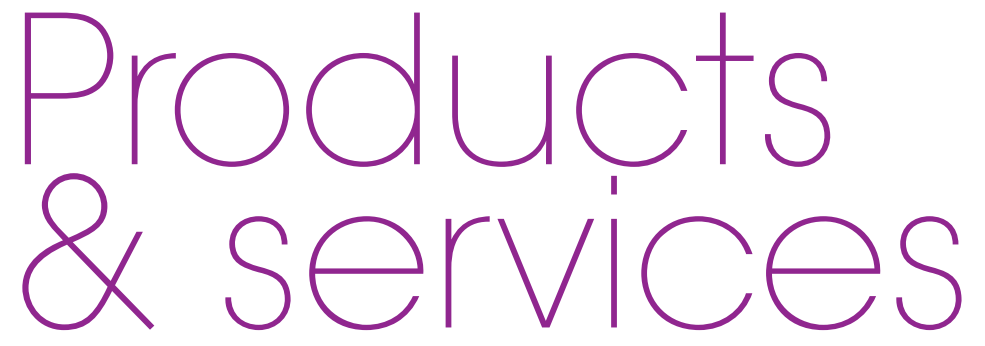

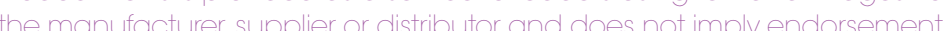 \\ by BDJ Team. Normal and prudent research \\ HOW MANY OF YOUR PATIENTS ARE SUFFERING IN SILENCE?
}

\author{
The GSK-supported ESCARCEL study, a \\ pan-European epidemiology study on \\ the prevalence of dentine hypersensitivity, \\ has revealed significant insights into the \\ condition. It exposed that more than two \\ out of five young adults in Europe suffer \\ from dentine hypersensitivity. Of those with \\ the condition, $28.4 \%$ said the pain was \\ important to them \\ Approximately $80 \%$ of sensitivity sufferers \\ are not currently using a desensitising \\ toothpaste. ${ }^{2}$ Over half of sufferers endure \\ the symptoms in silence and fail to seek \\ appropriate help from their dentist. \\ Research into patients' perspectives of \\ dentine hypersensitivity reveals that patients \\ experience a mixture of emotions about \\ it. Patients have reported feeling anxiety, \\ frustration and annoyance because of their \\ sensitive teeth. The condition can cause \\ sufferers to change their habits and avoid \\ foods or drinks they may otherwise enjoy. \\ There are too many patients that feel they \\ must "just get on with it". \\ It's important to recognise that some \\ patients are suffering and that your \\ recommendation could be their pathway
}

to minimising their symptoms. Use of

a desensitising toothpaste is a simple

yet effective way to manage dentine

hypersensitivity. Brushing twice daily should

already be part of a patient's oral health

care routine so swapping to a desensitising

toothpaste is an easy treatment plan

to follow.

Sensodyne products containing

NovaMin build a hydroxyapatite-like layer

over and within exposed dentine tubules, ${ }^{5}$ repairing dentine to provide clinically proven sensitivity relief. The robust layer binds firmly to collagen in dentine $e^{7.10}$ and resists daily oral challenges, 5,7,9,11,12 helping to protect against the pain of dentine hypersensitivity with twice daily brushing.

To request FREE trial sized packs for your practice visit wuw.gsk-dentalprofessionals. co.uk.

. West NX et al. J Dent 2013:41: 1-11

. GSK Data on File. March 2008.

3. Addy M. Int Dent J 2002; 52: 367-375.

4. Gibson B et al. Dentistry 2010; 1: 11-21

. Burwell A ef al. J Clin Dent 2010; 21 (Spec Iss): $66-71$

6. LaTorre G, Greenspan D C. J Clin Dent 2010; 21 (Spec Iss): 72-76.

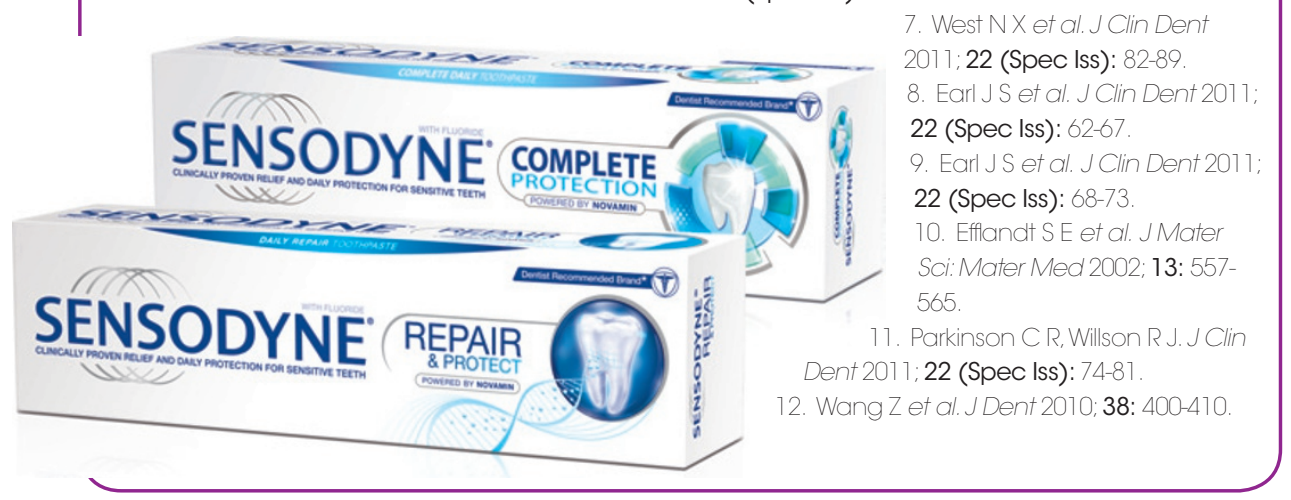

If you would like to promote your products or services direct to the dental industry in BDJ Team, just give Steve Brown a call on 02078434724 or drop an email to stephen.brown@nature.com.

\section{DELEGATES TEST DRIVE FIRST BLUETOOTH TOOTHBRUSH}

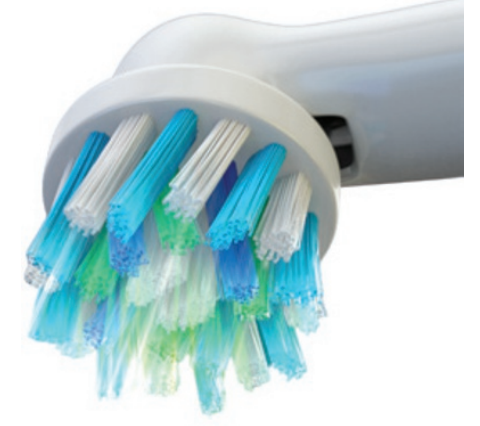

At this year's British Dental Conference and Exhibition in Manchester Oral-B introduced the new TEST DRIVE power brush trial programme; the SmartSeries electric toothbrush with Bluetooth 4.0 connectivity; and the CROSS ACTION brush head.

TEST DRIVE allows multiple users to experience Oral-B power and Oral-B toothpaste using a shared handle in a hygienic way. Oral-B has designed and produced specialised handles and refills that feature a Triple Protection-System: a sealing insert within the refill brush head works with the handle to help prevent saliva entering the handle; a specially designed protective covering works in combination with the sealing system to help provide extra protection against soling of the handle; and the cleaning and disinfection procedure ensures a hygienic next use.

The new CROSS ACTION brush head is Oral-B's most advanced power brush head to date that has perfectly angled bristles for a superior clean. Oral-B's new SmartSeries electric toothbrush is the world's first interactive electric toothbrush with Bluetooth 4.0 connectivity. The new toothbrush connects to the Oral-B App to provide real-time guidance while brushing - recording brushing activity as data - which patients can then share with their dental professional, helping to create a smarter, more personalised brushing routine.

The CROSS ACTION brush head will be available from July 2014 in the UK, and the SmartSeries electric toothbrush with Bluetooth 4.0 connectivity will be sold at limited retailers in the UK beginning in May. 